\title{
Degradation of plant cell wall polysaccharides by rumen bacteria and fungi
}

\author{
E Forano 1, V Broussolle 1, R Durand 2 \\ 1/NRA, Laboratoire de Microbiologie, C.R. de Clermont-Ferrand-Theix, 63122 Saint-Genès-Champanelle \\ 2 CNRS UMR 106 Centre de Génétique Moléculaire et Cellulaire, Biologie Cellulaire Fongique, Bt 405. \\ Université Lyon I, 69622 Villeurbanne Cedex, France
}

The rumen is inhabited by a dense and diverse microbial population consisting of obligate anaerobic bacteria, archaea, fungi and protozoa. The major structural polysaccharides of the plant cell wall become available as a source of energy to the host animal only through the activities of the rumen microflora. Cell walls consist of cellulose fibrils embedded in a matrix of other polysaccharides (hemicellulose and pectin) with varying amounts of lignification. Cellulose is the most widely distributed and abundant polysaccharide in plants. Although it is a linear polymer of glucose, its hydrolysis usually requires the combined action of different enzyme activities. The matrix polysaccharides vary in composition and structure. Xylan chains, for example, are substituted with arabinose and acetyl or methylglucuronic acid and enzymatic conversion of xylan to its monomeric components also requires the participation of several enzyme processes. In addition to cellulose, xylans and pectins, hemicelluloses such as xyloglucans, arabinans and $\beta 1,3$-glucans may be present together with protein material. Consequently, to degrade the plant cell wall, rumen microorganisms must produce a wide array of enzymes differring with respect to the bonds they cleave, their substrate specificities and their mode of action.

Gene cloning and DNA sequencing have enabled researchers to determine the amino acid sequences of many cellulases and xylanases from many different cellulolytic organisms. Analysis and comparison of the sequences have shown that the enzymes contain different domains: one or more catalytic domains, a cellulose-binding domain (CBD) and other domains that are usually involved in multi-enzyme complex (cellulosomes) formation or are of unknown function, usually separated by linkers (Gilkes et al, 1991). Genes concerned with plant cell wall polysaccharide breakdown have also been isolated from rumen bacteria and fungi, and their sequences have been determined, leading to a better understanding of the molecular organization of the enzymes and of the hydrolytic systems.

\section{Polysaccharidases of rumen bacteria}

Three rumen bacterial species are considered to be the major cellulolytic microorganisms in the rumen, Fibrobacter succinogenes, Ruminococcus albus and $R$. flavefaciens. These bacteria are able to grow efficiently on a wide range of forage material, as well as on purified cristalline cellulose. These species are also capable of solubilizing hemicelluloses. Some strains of Butyrivibrio fibrisolvens are also cellulolytic, but as they have only a limited ability to degrade purified or intact forage cellwall cellulose as well as having a higher xylanolytic activity, the major role of this species in the rumen is thought to be in xylan degradation (Dehority, 1993).

$F$. succinogenes is particularly active against crystalline cellulose, and many cellulases have been identified from this bacterium. However, cell-free extracts or isolated enzymes show almost no activity against this substrate, suggesting that some essential factor or enzyme organisation is lost in these extracts (Forsberg et al, 1994). Two endoglucanases ( $E G_{1}$ and $E G 2$ ), a cellobiosidase and a cellodextrinase have been purified from cultures of $F$. succinogenes $\$ 85$. EG2 was shown to possess separate catalytic and substrate-binding domains, however the gene has yet to be isolated. Genes coding for 2 other endoglucanases (EG3 and EGB) and for the previously purified cellodextrinase (CEDA) have been isolated from the same strain by cloning in $E$. coli and sequencing (Forsberg et al, 1994; Broussolle et al, 1994). EG3 and CEDA belong to family 5 , and EGB to family 9 of the glycosyl-hydrolases (Henrissat and Bairoch, 1993). A number of other genes 
coding for glucanase activities have been cloned in $E$. coli indicating that $F$. succinogenes $\mathrm{S} 85$ possesses at least nine distinct glucanase genes (Malburg and Forsberg, 1993). An additional 2 endoglucanase genes were cloned from other strains of $F$. succinogenes, and sequenced: EGA from the strain AR1, and EGC from the strain BL2. They both belong to family 9 (Cavicchioli et al, 1991; Bera et al, this issue). A mixed-linkage $\beta$-glucanase (lichenase) gene was also cloned from the strain S85 together with 4 distinct endoxylanase genes. The lichenase and 2 of the xylanase genes were sequenced. The endoxylanase XynB is a member of family 10 . The endoxylanase $\mathrm{XynC}$ consists of 2 catalytic domains belonging to family 11 and a third domain of unknown function (Forsberg et al, 1994). Two other xylanases have been purified from S85 cultures, but the genes have not been isolated. The sequences of all these genes indicated that, in addition to the catalytic domains, many of the enzymes carry other domains (EG3, $E G C$, the lichenase, $X y n C)$. Currently, the function of these domains is not known but they are not essential for the enzyme activity.

$F$. succinogenes possesses also a comprehensive set of xylan-debranching enzymes, including arabinofuranosidase, acetyl-xylan esterase, ferulic acid esterase and $\alpha$-glucuronidase (Forsberg et al, 1994).

The two other main cellulolytic rumen species, $R$. albus and $R$. flavefaciens, produce high molecular weight cellulolytic aggregates. Furthermore, cellulosome-like structures have been reported from these species (Stack and Hungate, 1984). Cellulases or their genes have been isolated from many different strains of the Ruminococci. From the strain F40 of $R$. albus, different cellulases have been purified, including a $\beta$-glucosidase, a cellobiosidase and two endoglucanases. A $\beta$-glucosidase gene and 6 cellulase genes have been isolated from 4 different strains of this species. All the cellulases belong to family 5 . $R$. flavefaciens FD1 exhibits many enzyme activities, including endo and exo-glucanase, cellodextrinase and laminarinase. These activities are associated in two complexes, each composed of numerous endoglucanases (Doerner and White, 1990). Seven cellulase genes have been isolated from 3 strains of $R$. flavefaciens. The enzymes belong to family 5 or 9 . In addition, 3 xylanase genes have been isolated from $R$. flavefaciens
F17, two of them are bifunctionnal: XynA possesses a family 11 catalytic domain and a family 10 catalytic domain, and XynD contains a family 11 catalytic domain and a family 16 lichenase domain (Flint et al, 1994).

Butyrivibrio fibrisolvens has been shown to carry two enzyme complexes on its surface, one having mainly xylanase activity and the other endoglucanase activity (Lin and Thomson, 1991). At least 11 different xylanases have been identified from this species. Genes coding for xylanase, xylosidase, endoglucanase and $\beta$-glucosidase have been cloned and sequenced. XylB has a bifunctional organization, consisting of both xylosidase and an arabinofuranosidase domains (Utt et al, 1991).

\section{The hydrolytic system of rumen anaerobic fungi}

Anaerobic fungi grow on a range of structural carbohydrates present in forage fiber. Rhizoids of vegetative thalli attack cell walls by penetration through the stomata and cracks in the epidermal layer. This phenomenon gives anaerobic fungi an advantage over bacteria in the degradation of recalcitrant tissues and reduces the textural strength of the plant material.

Although the primary structure of hydrolases from aerobic fungi and several prokaryotes have been analysed, few genes have been characterized from rumen fungi. The xylanase cDNA (xynA) isolated from $N$. patriciarum (Gilbert et al, 1992) was shown to contain two highly homologous catalytic domains. A second $C D N A$ encoding xylanase $B$ (XYLB) exhibited a N-terminal catalytic domain linked to a $\mathrm{C}$-terminal domain by a highly reiterated sequence (Black et al, 1994). The cDNA ceID encoding three multi-functional catalytic domains with endoglucanase, cellobio-hydrolase and xylanase activities has also been characterized (Xue et al, 1992). An endoglucanase gene (celB) isolated from $N$. patriciarum was shown to encode a modular protein: the catalytic domain was linked to a non-catalytic domain containing short tandem repeats (Zhou et al, 1994). Unlike the genes coding for aerobic fungal cellulases, celB was devoid of introns.

A multiprotein complex showing activity against crystalline cellulose and having no 
apparent xylanase activity has been found in $N$. frontalis (Wilson and Wood, 1992). Recently it has been reported that the major extracellular cellulase and hemicellulase activities of the anaerobic fungus Piromyces were associated with a cellulose-binding complex (Ali et al, 1995). A comparison of the enzymatic equipment of different rumen species, Neocallimastix frontalis, Piromyces rhizinflata and Sphaeromyces communis revealed that they exhibited the same range and nature of exo and endo-acting enzymes (Gomez de Segura et al, 1994). The enzymatic properties (optimum $\mathrm{pH}$ and temperature, $\mathrm{K}_{\mathrm{m}}$ ) were very similar from one enzyme's activity to another and from one species to another indicating that the different fungal species may fulfil similar roles in the rumen. $N$. frontalis isolated from sheep rumen was used as a model to clone the genes of the xylanolytic system. The secreted xylanolytic system of $N$. frontalis is characterized by a multiplicity of activities as revealed by zymogram analysis. Are these polypeptides the products of different xylanase genes or the result of post-translational modifications of the products of a limited number of genes? Two $\beta$-endoxylanases XYL1 and $X Y L 2$ with respective $\mathrm{Mr}$ of 45000 and 70000 have been purified and characterized from the culture medium of $N$. frontalis (Gomez de Segura and Fèvre, 1993). Both enzymes exhibited xylanase and carboxymethylcellulase activity indicating that they belong to the family 10 glycosidases (Henrissat and Bairoch, 1993). Different cDNAs designated xyn 3 and xyn 4 were isolated from an expression library of $N$. frontalis. Xyn3 was further characterized and was shown to contain a single open reading frame of $1821 \mathrm{bp}$ coding for a protein XYN3 of $\mathrm{Mr} 66000$. The predicted primary structure of XYN3 consisted of two large N-terminal reiterated regions of 223 amino acids with high homology $(88,3 \%)$. Each domain of XYN3, $X Y N 3 A$ and $X Y N 3 B$ showed significant homology with fungal and bacterial xylanases belonging to endoxylanase family 11. XYN3 and $X Y N 3 A$ were cloned into a bacterial expression plasmid harbouring a $6 \mathrm{His}$ $\mathrm{C}$-terminal tag and the recombinant proteins XYN3 and XYN3A purified from Escherichia coli. The recombinant proteins had a $\mathrm{Mr}$ of 66800 and 34000 respectively and hydrolysed xylan to xylo-oligosaccharides. Analysis of truncated forms of XYN3 confirmed that the full length protein contained two calalytic domains displaying similar substrate specificity. Western blot analysis using antiserum raised against XYN3 showed that the $N$. frontalis xylanase was not extensively glycosylated. XYN3 antiserum recognized similar polypeptides in the culture medium of two other rumen fungi Piromyces rhizinflata and Sphaeromyces communis.

Xyn4 was shown to contain a single open reading frame of $852 \mathrm{bp}$ coding for a predicted protein of $31.5 \mathrm{kDa}$ showing a high degree of identity with the xylanase XYNC from the rumen bacterium Fibrobacter succinogenes. A bacterial clone harboring XYN4 produces endoxylanase activity. Sequence comparisons and substrate specificity demonstrated that XYN4 also belongs to xylanase family 11 . However Northern analysis of total RNAs revealed a single transcript of $2.8 \mathrm{~kb}$ when using $X Y N 4$ as a probe, indicating that XYN4 is a truncated clone and that the native XYN4 protein consisted of more than one domain.

Biochemical and molecular studies demonstrated that the xylanase activities of $N$. frontalis are encoded by gene families. The production of active xylanases by $E$. coli shows that glycosylation is not required for enzyme activity. This makes it easier to screen expression libraries for new genes coding for plant cell-wall polysaccharide breakdown.

\section{Concluding remarks}

Despite the relatively high number of cellulases and cellulase genes isolated so far from rumen micro-organisms, none is highly active against crystalline cellulose. The ability of rumen bacteria and fungi to degrade ordered crystalline cellulose may involve the interaction of several enzymes, or their organization into high molecular weight complexes. Furthermore, few of the cellulase and xylanase genes that have been sequenced possess a substrate-binding domain. A sequence homologous to a cellulose-binding domain is present at the C-terminus of $B$. fibrisolvens End1 (Gilkes et al, 1991). Endoglucanase $E G 2$, purified from $F$. succinogenes, was found to possess a discrete cellulose binding domain, and two non-catalytic cellulose binding proteins have been purified from this species (Mitsumari and Minato, 1993; Forsberg et al, 1994). However the corresponding genes have not yet 
been cloned, and it is not known if these polypeptides belong to the known substratebinding domain families (Gilkes et al, 1991).

Several hemicellulases isolated from rumen microorganisms show more than one catalytic domain within the same polypeptide. One domain could be reiterated or the domains could be different and belong to the $F$ and $G$ xylanase families. It is not yet clear whether such an organization confers any catalytic advantage. In multifunctional enzymes, catalytic domains that differ in their specificities (xylanase, glucanase) might act synergistically on complex substrates. The modular structure of hemicellulase genes from rumen microorganisms could be explained by invoking gene duplications, recombination events between homologous genes or by postulating possible gene transfer between prokaryotes and eukaryotes living in the very dense resident microflora of the rumen.

Although many polysaccharidase genes have been characterized in recent years, the organization of the hydrolytic systems of the rumen micro-organisms is not known. Each bacterial or fungal species has been shown to possess many different cellulases and hemicellulases, belonging to a limited number of glysoside hydrolase families, and the role of each of these enzymes has still to be elucidated.

\section{Literature cited}

Ali BRS, Zhou L, Graves FM, Freedman RB, Black GW, Gilbert HJ, Hazlewood GP (1995) Cellulases and hemicellulases of the anaerobic fungus Piromyces constitute a multiprotein cellulose-binding complex and are encoded by multigene families. FEMS Microbio/ Lett 125, 1522

Black GW, Hazlewood GP, Xue GP, Orpin CG, Gilbert HJ (1994) Xylanase B from Neocallimastix patriciarum contains a noncatalytic 455-residue linker sequence comprised of 57 repeats of an octapeptide. Biochem $J 299$, 381-387

Broussolle V, Forano E, Gaudet G, Ribot Y (1994) Gene sequence and analysis of protein domains of $E G B$, a novel family $E$ endoglucanase from Fibrobacter succinogenes S85. FEMS Microbiol Lett 124, 439-448

Cavicchioli, East, Watson (1991) EndAFs a novel family $E$ endoglucanase gene from Fibrobacter succinogenes AR1. J Bacteriol 173, 3265-3268
Dehority BA (1993) Microbial ecology of cell wall fermentation. In: Forage cell wall structure and digestibility (Jung HG, Buxton DR, Hatfield RD, Ralph J, eds) ASA-CSSA-SSSA, Madison, USA, 425-453

Doerner KC, White BA (1990) Assessment of the endo- $\beta 1,4$-glucanase components of Ruminococcus flavefaciens FD1. Appl Environ Microbiol $56,1844-1850$

Flint HJ, Zhang JX, Martin J, Garcia-Campayo V, Daniel A, Wood TM (1994) Relationships of xylanase genes from rumen bacteria. In: Genetics, Biochemistry and Ecology of lignocellulose degradation (Shimada $\mathrm{K}$, Ohmiya K, Kobayashi Y, Hoshino S, Sakka K, Karita S, eds) Uni publishers Co, LTD, Tokyo, Japan, 188195

Forsberg CW, Gong J, Malburg LMJr, Zhu H, lyo A, Cheng KJ, Krell PJ, Phillips JP (1994) Cellulases and hemicellulases of Fibrobacter succinogenes and their role in fibre digestion. In: Genetics, Biochemistry and Ecology of lignocellulose degradation (Shimada K, Ohmiya K, Kobayashi $Y$, Hoshino S, Sakka K, Karita S, eds) Uni publishers Co, LTD, Tokyo, Japan, 125-136

Gilbert HJ, Hazlewood GP, Laurie JI, Orpin CG, Xue GP (1992) Homologous catalytic domains in a rumen fungal xylanase: evidence for gene duplication and prokaryotic origin. Mol Microbiol 6, 2065-2072

Gilkes NR, Henrissat B, Kilburn DG, Miller RCdr, Warren RAJ (1991) Domains in microbial $\beta-1,4-$ glycanases: sequence conservation, function, and enzyme families. Microbiol Rev 55, 303-315

Gomez de Segura B, Fèvre M (1993) Purification and characterization of two 1,4- $\beta$-xylan endohydrolases from the rumen fungus Neocallimastix frontalis. Appl Environ Microbiol $59,3654-3660$

Gomez de Segura B, Durand R, Rascle C, Fisseux C, Fèvre $M$ (1994) The xylanolytic system of rumen anaerobic fungi. In: Micro-organisms in Ruminant Nutrition (Prins RA, Stewart CS, eds) Nottingham University Press, 127-135

Henrissat B, Bairoch A (1993) New families in the classification of glycosyl hydrolases based an amino acid sequence similarities. Biochem $J 293$, 781-788

Lin LL, Thomson JA (1991) An analysis of the extracellular cellulases and xylanases of Butyrivibrio fibrisolvens H17C. FEMS Microbiol Lett 84, 197-204

Malburg LMJr, Forsberg CW (1993) Fibrobacter succinogenes $S 85$ possesses at least nine distinct glucanase genes. Can J Microbiol 39 , 882-891

Mitsumari M, Minato $H$ (1993) Purification of cellulose-binding proteins 1 and 2 from cell lysate 
of Fibrobacter succinogenes S85. J Gen Appl Microbiol 39, 361-369

Stack RJ, Hungate RE (1984) Effect of 3phenylpropanoic acid on capsule and cellulases of Ruminococcus albus 8. Appl Environ Microbiol 48, 218-223

Utt EA, Eddy CK, Keshav KF, Ingram LO (1991) Sequencing and expression of the Butyrivibrio fibrisolvens $x y l B$ gene encoding a novel bifunctional protein with $\beta$-D-xylosidase and $\alpha$-Larabinofuranosidase activities. App/ Environ Microbio/ 57, 1227-1234

Wilson CA, Wood TM (1992) The anaerobic fungus Neocallimastix frontalis: isolation and properties of a cellulosome-type enzyme fraction with the capacity to solubilize hydrogen-bond-ordered cellulose. App/ Microbiol Biotechnol 37, 125-129

Xue GP, Gobius KS, Orpin CG (1992) A novel polysaccharide hydrolase cDNA (celD) from Neocallimastix patriciarum encoding three multifunctional catalytic domains with high endoglucanase, cellobiohydrolase and xylanase activities. J Gen Microbiol 138, 2397-2403

Zhou L, Xue GP, Orpin CG, Black GW, Gilbert HJ, Hazlewood GP (1994) Intronless ce/B from the anaerobic fungus Neocallimastix patriciarum encodes a modular family $A$ endoglucanase. Biochem J 297, 359-364 